ESCENA

Revista de las artes
Publicación semestral. ISSN 2215-4906

Volumen 77 - Número 2

Enero - Junio 2018

\title{
Orquesta Sinfónica de Galicia, estudio sobre su programación en el período 1992-2002
}

The Orquesta Sinfónica de Galicia, research on its programming in the period 1992-2002

Aránzazu García Escuredo

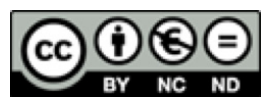

Esta obra está bajo una licencia Creative Commons Reconocimiento-No comercial-Sin Obra Derivada 


\title{
Orquesta Sinfónica de Galicia, estudio sobre su programación en el período 1992-2002
}

\author{
The Orquesta Sinfónica de Galicia, research on its \\ programming in the period 1992-2002
}

\author{
Aránzazu García Escuredo ${ }^{1}$ \\ Universitat Politècnica de València (UPV) \\ España
}

Recibido: 24 de mayo de 2017 Aprobado: 20 de junio de 2017

\section{Resumen}

La Orquesta Sinfónica de Galicia (OSG) está próxima a celebrar su veinticinco aniversario. En ese tiempo, ha pasado de ser una orquesta pionera en su área a competir con otras entidades en la oferta musical, a un público cada vez más formado, informado y diversificado. A través del análisis del repertorio interpretado en los primeros diez años de existencia de la OSG, la autora busca determinar el perfil propio que la OSG se ha ido creando frente a un público específico para llegar a consolidar su posición como referente orquestal a nivel nacional. El elemento diferenciador más importante es la labor que la OSG ha realizado en la recuperación, puesta en valor y promoción de las obras de compositores gallegos, pero también es relevante el papel de la OSG en el incremento de la oferta cultural en La Coruña al ofrecer por primera vez una programación estable de música clásica.

Palabras clave: orquesta; repertorio; programación musical; Galicia; música clásica

1 Máster en Artes Escénicas, Universidad de Vigo, España. Doctorando en la Universitad Politécnica de València (UPV), en el mismo país. Correo electrónico: arantxaescuredo@gmail.com 


\begin{abstract}
The Orquesta Sinfónica de Galicia (OSG) is about to celebrate its twenty-fifth anniversary. In that time, it has passed from being a pioneering orchestra in its area to compete with other entities in order to offer to a more and more informed audience to an audience more and more formed, informed and diversified Through the analysis of the repertoire interpreted in the first ten years, the author seeks to determine the profile that the OSG has been creating for a specific audience in order to consolidate its position as a national orchestral. The most important differentiating element is the work that OSG has done in the recovery, enhancement and promotion of the works of Galician composers, but also the role of the OSG in increasing the cultural activities programmed in La Coruña by offering for the first time a stable classical music program.
\end{abstract}

Keywords: orchestra; repertory; musical programming; Galicia; classical music 


\section{Introducción}

La década de 1990 fue una época de fundación de nuevas Orquestas Sinfónicas por todo el territorio español. Entre los años 1987 y 2000 se fundaron en España: la Orquesta de la Comunidad de Madrid (1987), la Orquesta Sinfónica del Vallés (1987), la Orquesta Sinfónica de Baleares "Ciudad de Palma" (1988), La Orquesta de Cadaqués (1988), la Orquesta Sinfónica de Sevilla (1990), la Orquesta Ciudad de Granada (1990), La Orquesta Sinfónica de Castilla y León (1991), la Orquesta del Principado de Asturias (1991), la Orquesta de Córdoba (1992), la Orquesta Sinfónica de Galicia (1992), la Joven Orquesta Nacional de Cataluña (1993), la Real Filharmonía de Galicia (1996), la Orquesta Oviedo Filarmonía (1999), o la Orquesta de Extremadura (2000), entre otras (Asociación Española de Orquestas Sinfónicas (AEOS), s.f.).

En Galicia, la Orquesta Sinfónica de Galicia (OSG) fue la pionera y situó a esta comunidad, en palabras de su ex-director Víctor Pablo Pérez, "en el mapamundi de la música clásica, un sector muy exigente" (Nebreda, 28 de agosto del 2011). Veinticinco años después, la OSG ha visto surgir otras numerosas agrupaciones, muchas de ellas formadas por sus propios integrantes. Desde ensambles y orquestas de cámara: Zoar ensemble (2009), Grupo instrumental s. XX (1996), la Orquesta de Cámara de la OSG (1995) hasta otras Orquestas de diversa consideración y, esencialmente, ajenas a la plantilla de la propia Orquesta Sinfónica de Galicia, como la Orquesta Sinfónica de Melide (1993), la Orquesta de Cámara Gallega (1995), la Orquesta Sinfónica Vigo 430 (2005), la Orquesta Gaos (2009), entre otras.

El objetivo de este artículo es vislumbrar la orientación de la programación musical en los primeros años de la OSG a través del análisis de las obras interpretadas en dichos programas. Se trata de determinar las líneas que la Orquesta ha seguido en la elección del repertorio a interpretar en función del público, las posibilidades de la plantilla, las circunstancias económicas y también la intención de otorgar una personalidad propia y diferenciadora a la orquesta.

\section{Metodología}

Para la realización de este estudio se han manejado las programaciones completas de las temporadas 1992-1993 a 2002-2003 publicadas cada nueva temporada por el Consorcio para la Promoción de la Música. Aquellos datos que no constaban en dichas programaciones (como los casos en los que el repertorio o los intérpretes estaban por determinar en el momento de la impresión de los folletos) fueron facilitados por la propia oficina de la Orquesta a partir de la información que constaba en sus archivos. En dichos programas figuran, no solamente las fechas, lugares, repertorio, solistas y director, sino también otros datos relevantes como las entidades colaboradoras, los patrocinios y la plantilla titular de cada temporada. 


\section{El ambiente musical en La Coruña antes de la OSG}

La ciudad de La Coruña, sede de la OSG, tiene una historia irregular en lo que se refiere a la existencia de agrupaciones orquestales en los años anteriores a la fundación de la misma. En los primeros años del siglo XX, se funda la Sociedad Filarmónica de La Coruña (17 de mayo de 1904). Sociedad que, además de promover la celebración de conciertos, intervino en la creación de una Agrupación de Instrumentos de Arco en 1916, que devendría en la Orquesta Filarmónica Coruñesa en 1934, ya como Orquesta sinfónica. Dicha entidad mantuvo una programación regular de conciertos con una media de alrededor de diez conciertos al año entre 1920 y 1980. A esta actividad de la Sociedad Filarmónica de La Coruña hay que añadir las visitas anuales de la Orquesta Sinfónica de Madrid, entre 1920 y 1936, y la Orquesta Municipal de Bilbao, entre 1946 y 1962 (Alén, 2000). "La Sociedad Filarmónica de La Coruña promueve conciertos de corte tradicional (sobre todo, de música 'clásica') pero de gran arraigo entre la población coruñesa" (Alén, 1997, p.18). La promoción del repertorio lírico en esta época quedaba casi, exclusivamente, a cargo de la Asociación de Amigos de la Ópera, fundada en 1952 y responsable del Festival de Ópera de La Coruña casi ininterrumpidamente desde 1953. Con el paréntesis obligado de la Guerra Civil y la Postguerra hubo un largo periodo de esterilidad, con la salvedad de los intentos por mantener una agrupación estable de la Banda-Orquesta Municipal. Hasta que en 1972 se creó la Orquesta de La Coruña, dirigida por Rogelio Groba. Diez años después, vino a ser sustituida por la Orquesta de Cámara Municipal, con el mismo director. En 1992, el Consorcio para la Promoción de la Música (creado un año antes), dio los pasos para constituir la Orquesta Sinfónica de Galicia (Carreira, 1994).

\section{Los factores que intervienen en la programación musical}

Una definición de qué es la programación musical podría resumirse diciendo que es la elección coherente y atractiva de intérpretes y de obras acorde a las posibilidades económicas y al perfil de la institución, así como a los tipos de público destinatarios ... Programar es, en esencia, un proceso de toma de decisiones que viene condicionado por cinco variables principales: el repertorio, el intérprete, el presupuesto, el mercado y el público (Marín, 2013, p. 88).

Parece haber un acuerdo en la intervención de diversos factores confluyentes en la elección del repertorio de una orquesta sinfónica. La programación ha de satisfacer al público, pero también ha de corresponderse con las posibilidades económicas reales de la institución, así como la disponibilidad de los intérpretes y la adecuación de la plantilla. "La programación aparece, así como la materialización de todos los vectores que confluyen en la sala de conciertos: música, músicos, público, patrocinadores y demás fuerzas vivas del entorno" (Rodríguez Suso, 2003, p. 261). Programar obras que por fecha ya están libres de derechos de autor o resultan más baratos, también es un factor que muchas orquestas tienen en cuenta y 
que figura entre los motivos por los que la música sinfónica más actual se programa con menos frecuencia. Aunque varía de país a país, por regla general los derechos de autor expiran entre 50 y 70 años posteriores al fallecimiento del autor ${ }^{2}$.

La OSG nace en el año 1992, donde confluyen las celebraciones a nivel nacional del $\checkmark$ Centenario del descubrimiento de América, las Olimpiadas de Barcelona y la Exposición Internacional de Sevilla, pero a la vez, la entrada en vigor del tratado de Maastrich que sienta las bases de la Unión Monetaria Europea. Un periodo en el que las instituciones realizan enormes inversiones para mejorar la imagen del país y para la promoción del mismo en el extranjero y donde, sin embargo, la realidad económica nacional se dirige hacia una importante recesión que gestada a mediados de 1992 tendría sus momentos más graves en 1993 (Gil-Ruiz \& Iglesias, 2007; Centro de Estudios del Cambio Social, 1994).

La financiación de las orquestas implica estructuras de una gran complejidad. Entre otros muchos criterios, pueden clasificarse dichas entidades en orquestas de financiación autonómica, local, ambas o exclusivamente nacional. Por su parte, la OSG es una orquesta de financiación mixta (Arboleya Montes, 2014). Durante su historia, la OSG ha contado con el patrocinio del Ayuntamiento de la Coruña y el apoyo y la colaboración de la Diputación de La Coruña, la Xunta de Galicia, la Sociedad de Amigos de la Ópera y la Fundación Caixa Galicia, principalmente, amén de patrocinios y mecenazgos puntuales de fundaciones, empresarios y negocios locales para ciertos conciertos, ciclos de conciertos o eventos concretos. Existe, además, un sistema de micro mecenazgos a cargo de particulares.

La primera plantilla de la OSG contó con setenta y tres músicos, quince de ellos españoles y la cifra subió a ochenta y nueve músicos al año siguiente. Constatamos un cierto grado de permanencia y estabilidad en la plantilla, a pesar de los diversos ajustes a lo largo de los años. Especialmente, con el primer relevo de la dirección a manos de Víctor Pablo Pérez en sustitución de Sabas Calvillo, tan solo un año después de la fundación de la Orquesta. Esa relativa estabilidad, junto con la permanencia de una misma dirección en un lapso largo, ha configurado un repertorio propio con un sonido característico y permite vislumbrar una tendencia en cuanto a la elección de autores y obras. En lo que respecta al público, hay que tener en cuenta lo señalado en relación al ambiente musical previo a la OSG, no solo en La Coruña, sino también en la comunidad gallega. El surgimiento de la Real Filharmonía y otras orquestas en un periodo de pocos años posterior a la fundación de la OSG, es una significativa muestra de la demanda del público por este tipo de oferta musical.

2 Por ejemplo, la legislación española contempla en el artículo 26 del REAL DECRETO LEGISLATIVO 1/1996, de 12 de abril, por el que se aprueba el texto refundido de la Ley de Propiedad Intelectual, una duración de setenta años para los derechos posteriores al fallecimiento del autor. 


\section{La programación musical de la OSG entre 1992 y 2002}

Si nos circunscribimos a los conciertos sinfónicos sin tener en cuenta los conciertos escolares ni las representaciones operísticas, la media anual de conciertos ofrecidos por la OSG ronda los cincuenta y ocho por año, con unos treinta y cinco programas diferentes de media.

\begin{tabular}{|c|c|c|}
\hline \multicolumn{3}{|c|}{$\begin{array}{l}\text { Cuadro 1. Relación entre número de } \\
\text { conciertos y programas interpretados } \\
\text { por la OSG en la década 1992-2002 }\end{array}$} \\
\hline Temporada & $\begin{array}{l}\text { Número de } \\
\text { conciertos }\end{array}$ & Programas \\
\hline 1992-93 & 32 & 18 \\
\hline 1993-94 & 56 & 31 \\
\hline $1993-94$ & 69 & 39 \\
\hline $1995-96$ & 67 & 45 \\
\hline $1996-97$ & 66 & 42 \\
\hline $1997-98$ & 65 & 47 \\
\hline 1998-99 & 66 & 43 \\
\hline $1999-00$ & 48 & 28 \\
\hline $2000-01$ & 53 & 31 \\
\hline 2001-02 & 61 & 33 \\
\hline Media & $58^{\prime} 3$ & $35^{\prime} 7$ \\
\hline \multicolumn{3}{|c|}{ Fuente: Elaboración propia. } \\
\hline
\end{tabular}

Gráfico 1. Relación entre número de conciertos y programas interpretados por la OSG en la década 1992-2002

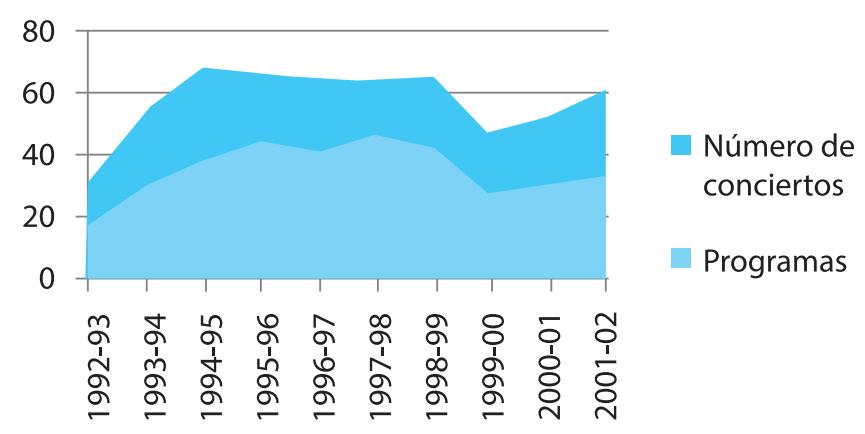

Fuente: Elaboración propia

Los conciertos didácticos añaden a ese total alrededor de cuatro programas al año desde la temporada 1993-94, con una media anual de doce conciertos didácticos por temporada. Seguidos, generalmente, por un Concierto en familia con el mismo programa. La regularidad e importancia que los conciertos didácticos han adquirido a lo largo de los años demuestra una vocación de la OSG por formar nuevo público, que ha evolucionado con la introducción de conciertos preescolares y conciertos didácticos para sordos; esfuerzo que queda patente con el encargo de obras específicamente para dicha finalidad ( $\mathrm{O}$ coelliño branco rebranco y el Concierto de los sentidos, de Botella y Verdú, respectivamente).

En cuanto al repertorio operístico, la OSG ha participado, principalmente, en dos eventos anuales: el Festival de Ópera de La Coruña, organizado por la Asociación de Amigos de la Ópera de La Coruña, que suele tener lugar en septiembre, y el Festival Mozart, que desde 1998 se celebraba en la Coruña hasta su reciente cancelación en 2014, que tenía ocasión en torno a mayo y junio. En este contexto, Verdi, Puccini y Mozart son los autores más interpretados en esta década, seguidos por Rossini. 


\begin{tabular}{|c|c|c|c|}
\hline \multicolumn{4}{|c|}{ Cuadro 1. Óperas completas interpretadas } \\
\hline $\begin{array}{l}\text { Festival de Ópera de } \\
\text { La Coruña }\end{array}$ & $\begin{array}{c}\text { Óperas entre } \\
1992-2002\end{array}$ & Festival Mozart & $\begin{array}{c}\text { Óperas entre } \\
1998-2002\end{array}$ \\
\hline Verdi & 4 & Mozart & 4 \\
\hline Puccini & 4 & Rossini & 3 \\
\hline Donizetti & 2 & Haydn & 1 \\
\hline Massenet & 2 & Beethoven & 1 \\
\hline Offenbach & 1 & Salieri & 1 \\
\hline Mozart & 1 & & \\
\hline Bizet & 1 & & \\
\hline Gounod & 1 & & \\
\hline \multicolumn{4}{|c|}{ Fuente: Elaboración propia. } \\
\hline
\end{tabular}

En esto, se sigue la tendencia más ortodoxa en cuanto a repertorio operístico:

En el caso de la ópera, en torno al 75\% de las óperas programadas en una temporada en los principales teatros del mundo pertenecen a solo diez compositores: Mozart, Verdi, Puccini, Wagner, Rossini, Donizetti, Richard Strauss, Bizet, Janacek y Händel. Es más, solo los cuatro primeros autores ya superan el 50\% de las obras programadas (Marín, 2013, p. 92).

La Zarzuela también ha ocupado un lugar importante en la programación de la Orquesta, ya que es un repertorio que siempre tiene una buena acogida entre el público en general. Chapí y Chueca son los compositores de Zarzuela más interpretados en este periodo. Ambos ocupan un lugar importante también en la lista general de autores, donde se encuentran situados en el puesto vigesimocuarto y vigesimosexto de la totalidad de compositores interpretados de todos los géneros y en los puestos tercero y cuarto, respectivamente, de entre los compositores españoles. La Zarzuela más interpretada es en estos años La Gran Vía, seguida por La Revoltosa. La atención que la OSG ha prestado a la Zarzuela, también se evidencia en la realización de una grabación de La tabernera del Puerto de Sorozábal en la temporada 1995-96, con María Bayo y Plácido Domingo en los roles principales. La presencia de María Bayo ha sido muy habitual en la programación de la Orquesta durante estos años.

Si atendemos al total de intervenciones de la OSG en estos años tenemos dos mil ciento cincuenta y dos obras interpretadas por la orquesta, de un total de doscientos cuarenta y ocho compositores diferentes. Cabe destacar que, en ese listado, la orquesta solo ha interpretado en dos ocasiones un programa con la obra de una compositora, Ofertorium de Sofía Gubaidulina, ella ha sido la única mujer incluida en la programación de toda una década, lo que representa el $0.4 \%$ del total de compositores y el $0.09 \%$ de las obras. 
Al margen de lo anterior, la OSG ha interpretado todos los años un concierto especial de fin de año, al estilo del tradicional concierto de Año Nuevo que ofrece la Filarmónica de Viena, con un repertorio de Valses y Polkas, principalmente, de la familia Strauss. También ha ofrecido conciertos de carácter más alejado del repertorio académico con música popular como bandas sonoras y música pop, con la intervención en muchos casos de músicos de música moderna sobradamente conocidos por la audiencia (Serrat, Juan y Junior, J. M. Cano). La mayor parte de este tipo de conciertos han sido realizados fuera de su sede y en el marco de eventos festivos puntuales, como las fiestas de María Pita. Una manera de dar a conocer la Orquesta al público menos acostumbrado a la música académica o aquel que, a priori, siente rechazo hacia la música sinfónica. En el siguiente cuadro pueden verse los diez compositores más interpretados por la OSG en este periodo. Resulta muy llamativo que entre Mozart y el siguiente compositor más interpretado haya una diferencia de casi sesenta piezas.

\begin{tabular}{|c|c|c|c|}
\hline Orden & Autor & Veces interpretado & $\%$ \\
\hline $1^{\circ}$ & Mozart & 157 & $7 ’ 2 \%$ \\
\hline $2^{\circ}$ & Beethoven & 98 & $4^{\prime} 5 \%$ \\
\hline $3^{\circ}$ & Brahms & 87 & $4 ' 04 \%$ \\
\hline $4^{\circ}$ & Tchaikovski & 73 & $3,3 \%$ \\
\hline $5^{\circ}$ & Schubert & 54 & $2 ' 5 \%$ \\
\hline $6^{\circ}$ & Falla & 49 & $2{ }^{\prime} 2 \%$ \\
\hline $7^{\circ}$ & R. Strauss & 43 & 1'99\% \\
\hline $8^{\circ}$ & Shostakovich & 39 & $1 ' 81 \%$ \\
\hline $9^{\circ}$ & Wagner & 39 & $1 ' 81 \%$ \\
\hline $10^{\circ}$ & Dvorak & 38 & $1{ }^{\prime} 76 \%$ \\
\hline
\end{tabular}

En el Gráfico 2 (disponible en la página siguiente) pueden verse, asimismo, la totalidad de los compositores interpretados de mayor a menor. Se han excluido aquellos que lo fueron en menos de diez ocasiones para facilitar la visualización. De los compositores nacionales, es Falla el compositor que más veces ha sido interpretado por la OSG. Sin embargo, es destacable la especial atención que la Orquesta ha prestado a los compositores gallegos (nacidos o que desarrollaron su trabajo en Galicia). En este terreno, la OSG ha llevado a cabo una labor consciente de recuperación y puesta en valor de este acervo de obras. Hechos que, la propia Orquesta se encarga de destacar en varios de los programas anuales de temporada editados por el Consorcio para la Promoción de la Música, en los que destaca, especialmente, los autores gallegos interpretados cada temporada. Las temporadas 
1993-1994 y 1994-1995 contaron con la mayor presencia de compositores gallegos que tienen en su programación. Apenas al comienzo de su historia, la OSG ya marca una clara intención de servir de vehículo para recuperar y dar visibilidad a la composición gallega.

Gráfico 2. Compositores más interpretados por la OSG

\section{Obras interpretadas, por autores}

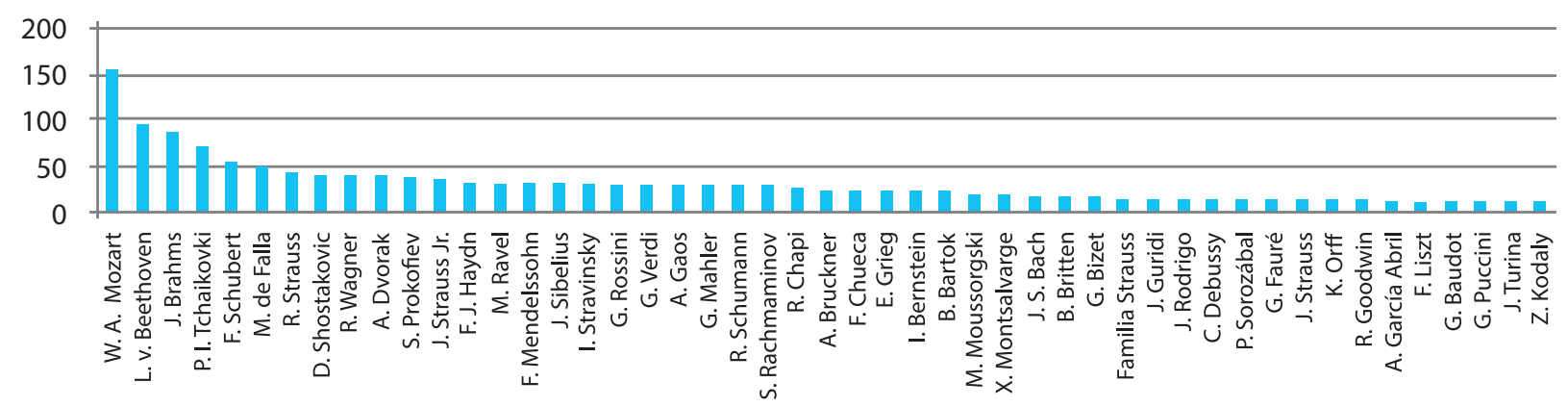

Fuente: Elaboración propia Gráfica a partir de los datos extraídos de las programaciones anuales de la OSG

La música gallega ha estado abandonada durante décadas y su olvido ha sido generalizado, tanto fuera, como dentro de Galicia. "Resulta llamativa la falta de referencias a la música y a los músicos gallegos en enciclopedias, diccionarios y estudios más especializados realizados en el curso de este siglo" (Alén, 1997, p.5). Así pues, este interés de la OSG por programar obras de compositores gallegos supone una importante aportación a esta labor de recuperación, que comenzó a manifestarse en diversas entidades académicas como la Universidad de Santiago, e institucionales como el IGAEM o el Consello da Cultura Galega a finales del siglo XX. De los dieciocho compositores gallegos interpretados por la OSG en este periodo, once son compositores nacidos en pleno siglo XX, de ellos, nueve nacieron en torno a mediados de siglo. En el tercer tercio de dicho siglo, se fomenta en Galicia la creación musical que, en cuestiones estilísticas, pivotará entre la referencia a la música popular autóctona y cierto universalismo con tendencia hacia lenguajes más modernos (López Cobas, 2013). La mayor parte de estos compositores son una muestra representativa de ambas tendencias (veáse Cuadro 3).

Es, sin duda, Gaos el compositor gallego que más relevancia ha tenido en la programación de la Orquesta Sinfónica de Galicia en el periodo 1992-2002. Su presencia en los programas de la orquesta es regular y frecuente. Esto lo convierte en un compositor de peso en el repertorio de la orquesta, no solamente si atendemos al número y frecuencia de su aparición en programa, sino también al tomar en consideración que es el segundo compositor español más interpretado por la OSG después de Falla. A todo lo anterior, hay que añadir que la Orquesta ha realizado la grabación de dos discos compactos de este autor. 


\begin{tabular}{|c|c|c|}
\hline \multicolumn{3}{|c|}{ Cuadro 3. Relación de autores gallegos y obras interpretadas } \\
\hline Autor & Obras & Veces interpretado \\
\hline Gaos (1874-1959) & 5 & 26 \\
\hline Baudot (1884-1938) & 2 & 10 \\
\hline Groba (1930- ) & 5 & 9 \\
\hline López García-Picos (1922-2009) & 4 & 8 \\
\hline Balboa (1958-2004) & 5 & 7 \\
\hline Macías Alonso (1958-1995) & 3 & 7 \\
\hline De Paz (1963- ) & 2 & 7 \\
\hline Vara (1959 -) & 3 & 5 \\
\hline Vila (1943- ) & 2 & 4 \\
\hline Rodríguez Losada (1886-1973) & 2 & 4 \\
\hline Soutullo (1880-1932)-(Groba) & 1 & 3 \\
\hline Durán (1960- ) & 1 & 2 \\
\hline Montes (1840-1899)-(Groba) & 1 & 2 \\
\hline Pereiro (1957- ) & 1 & 2 \\
\hline Veiga (1842-1906)-(Groba) & 1 & 2 \\
\hline Viaño (1960-1991) & 1 & 1 \\
\hline Kress (1964- ) & 1 & 1 \\
\hline Del Adalid (1844-1881) & 1 & \\
\hline Fuente: Elaboración propia. & & 2 \\
\hline
\end{tabular}

Además, con la creación de la Orquesta Sinfónica de Galicia en 1992, el movimiento de recuperación del compositor se vio incrementado, pues dicha agrupación se ha ocupado en numerosas ocasiones de la difusión y grabación de la música de Gaos. Muestra de ello es la grabación integral de su obra sinfónica, lo que fomentó, sino de forma directa, la edición de partituras (Painceira Luaces, 2013, p. 22).

Existen, también, otros dos discos compactos grabados en esta década, dedicados, respectivamente, a otros dos compositores gallegos: Balboa y Macías Alonso. Del total de piezas que se han interpretado, se han llevado al escenario ciento veintisiete conciertos diferentes para varios instrumentos, esencialmente, piano y violín. Los más numerosos son los conciertos para piano, en lo que influye, sustancialmente, la participación de la OSG en el Concurso Internacional de Piano "Ciudad de Ferrol". Sigue el violonchelo, con mayor número de conciertos, seguido del clarinete, pero también programan conciertos para flauta, guitarra, oboe, trompa, trompeta, fagot o arpa. 
En cuanto a las sinfonías, se han interpretado un total de ciento cuarenta y siete, pero aquí se incrementa el número de veces que se han repetido las mismas obras en diversos programas. Algo lógico si tenemos en cuenta que el caché del intérprete solista de los conciertos incrementa el gasto necesario para poner una obra en programa. De todas las obras interpretadas por la OSG, es la Sinfonía No 9 en Sol Mayor "del Nuevo Mundo" de Dvorak la que más veces ha aparecido en programa, con un total de catorce ocasiones; seguida de la Impresión Nocturna de Gaos, con trece ocasiones y una grabación. El mismo número de veces que se han interpretado las dos suites orquestales de El Sombrero de Tres Picos de Falla. El Carmina Burana de Orff le sigue con doce interpretaciones, las mismas que la Sinfonía No 29 en La Mayor KV 186a (201) de Mozart.

Es muy habitual encontrar programas en los que aparecen obras más o menos contemporáneas o de autores menos escuchados, el resto del programa suele recurrir a las obras más conocidas y canónicas de compositores ampliamente conocidos por el público. Por ejemplo, La cena de las cenizas de Sotelo acompañado por la Sinfonía No 7 en la mayor; Op. 92 de Beethoven, o el Concierto para arpa y oboe de Henze entre La vida breve de Falla y El mar de Debussy, o El mito finlandés de Rautavaara antes de la Sinfonía No 4 en la mayor, Op. 90, la "Italiana", de Mendelssohn.

Las estadísticas que la Sociedad General de Autores y Editores (SGAE) y el Ministerio de Educación, Cultura y Deporte publican regularmente sobre hábitos de consumo cultural, muestran que Galicia ha experimentado un aumento significativo de asistencia a conciertos de música clásica desde 1999 hasta 2003. Asimismo, el Observatorio da Cultura Galega muestra un aumento significativo del número de conciertos de música sinfónica en Galicia, que pasó de apenas 33 en 2001 a 125 en 2004.

\section{Conclusiones}

La Orquesta Sinfónica de Galicia aparece en el panorama musical gallego en un periodo en el que la oferta sinfónica no cubría suficientemente la demanda del público. El surgimiento de diversas agrupaciones sinfónicas en un periodo breve de tiempo posterior a la OSG parece corroborar el interés del público por este tipo de música. Aunque la OSG apareció en un periodo económicamente complicado para el país, el apoyo institucional y privado le permitió salir adelante con una programación cada vez más ambiciosa.

Desde los primeros años, la OSG ha invertido esfuerzos en la captación y en la formación de nuevo público, bien a través de los conciertos didácticos, bien dándose a conocer en eventos de carácter más popular fuera de su sede (conciertos dentro de la programación de fiestas del Ayuntamiento de La Coruña). Tanto con repertorios alejados de lo habitual en una Orquesta Sinfónica, como bandas sonoras o los conciertos sobre repertorio 
popular o con músicos de música moderna o pop, como Serrat o Cano, Juan y Junior, entre otros. El incremento del número de conciertos sinfónicos en la comunidad y el aumento del interés general por la música clásica que reflejan las encuestas sobre hábitos culturales parecen corresponder con este esfuerzo.

La elección del repertorio a lo largo de estos primeros diez años se ha orientado, esencialmente, hacia obras y autores bien conocidos por el público y con un peso importante dentro del llamado canon de la música académica. Una programación tradicional para un público poco habituado a novedades. La introducción de obras y autores menos conocidos se ha realizado de forma esporádica y siempre con la precaución de integrar dichas novedades en programas más familiares para el público. A pesar de lo anterior, la OSG ha apostado por los compositores gallegos y por la recuperación y puesta en valor de sus obras desde sus inicios. Un trabajo realizado conscientemente, no solo con la interpretación de sus obras, sino con la realización de grabaciones, estrenos absolutos e incluso encargos. Gaos ha sido el compositor gallego más beneficiado por esta política de recuperación de la Orquesta.

\section{Referencias}

Alén, $M^{2}$. P. (1997). Panorama y reflexiones sobre un cuarto de siglo de historiografía y actividades musicales en Galicia (CA. 1972-1997). AEDOM: Boletín de la Asociación Española de Documentación Musical. 4(2), 5-69.

Alén, Ma. P. (octubre 2000). Reflexiones sobre el ambiente musical en La Coruña (1920-1980) a través del "Fondo Bugallal" (213-234). Campos interdisciplinares de la musicología: V Congreso de la Sociedad Española de Musicología Lolo. Barcelona, España.

Arboleya Montes, I. (2014). Orquestas Sinfónicas: caída y auge. (Trabajo de Fin de Máster). Instituto Complutense de Ciencias Musicales Máster en Gestión Cultural: Música, Teatro y Danza, España.

Asociación de Amigos de la Ópera de A Coruña. Festivales. Recuperado de: http://www. amigosoperacoruna.org/la-asociación/

Asociación Española de Orquestas Sinfónicas. (AEOS). Orquestas asociadas. Recuperado de: http://www.aeos.es/orquestas.htm.

Carreira, X. M. (1994). 200 años de Orquestas coruñesas (16-21). Orquesta Sinfónica de Galicia, Grandes Conciertos de Palacio, I Festival de Música de La Coruña 1994-95.

Centro de Estudios del Cambio Social. (Ed.). (1994). España 1993, una interpretación de su realidad social. Madrid: Fundación Encuentro. 
Consello da Cultura Galega. Observatorio da cultura Galega. Recuperado de: http://observatorio.consellodacultura.gal/coxunturas/texto?id=859

Gil-Ruiz, C.L \& Iglesias, J. (2007). El gasto público en España en un contexto descentralizado. Presupuesto y Gasto Público 47, 185-206.

López Cobas, L. (2013). Historia da Música en Galicia. Sarria, Lugo: Ouvirmos.

Marín, M. A. (2013). Tendencias y desafíos de la programación musical. Brocar. Cuadernos de Investigación Histórica. 37, 87-104.

Ministerio de Educación, Cultura y Deporte (MECD). Resultados de la Encuesta de Hábitos y Prácticas Culturales en España. Recuperado de: http://www.mecd.gob.es/prensa-mecd/actualidad/2015/09/20152509-encuesta.html.

Nebreda, M. (28 de agosto del 2011). La joven orquesta que situó a Galicia en el mapa de la música clásica. El Mundo. Recuperado de: www: http://www.elmundo.es/elmundo/2011/08/27/galicia/1314467852.html.

Painceira Luaces, V. (2013). La revalorización de la figura de Andrés Gaos en Galicia. (Tesis doctoral). Universidad de Oviedo, España.

Rodríguez Suso, C. (2003). Ochenta años programando música: la gestión de contenidos en la Orquesta de Bilbao. En Rodríguez Suso (Ed.), Bilbao Orkestra Sinfonikoa / Orquesta Sinfónica de Bilbao. Ochenta años de música urbana 1922-2001 (Tomo I, pp. 259365). Bilbao: Billbao Bizkaia Kutxa.

Sociedad General de Autores y Editores (SGAE). Anuarios de las artes escénicas, musicales y audiovisuales. Recuperado de: http://www.anuariossgae.com/home.html

Sociedad Filarmónica de La Coruña. Historia. Recuperado de: http://www.filarmonicacoruna.com/historia/ 